\title{
Diagnostic impact of signs and symptoms in acute infectious conjunctivitis: systematic literature search
}

\author{
Remco P Rietveld, Henk C P M van Weert, Gerben ter Riet, Patrick J E Bindels
}

In acute infectious conjunctivitis the general practitioner has to distinguish a bacterial cause from a viral one to select the patients who are most likely to benefit from antibiotic treatment. The doctor makes this distinction on the basis of the presenting signs and symptoms. Additional diagnostic investigations, such as a culture of the conjunctiva, are seldom done, mainly because the result is delayed a few days.

Most treatment trials show that a bacterial pathogen can be isolated from the conjunctiva in only half of patients with clinically diagnosed acute bacterial conjunctivitis. However, general practitioners prescribe antibiotics in most cases of acute infectious conjunctivitis. Although the subject has never been investigated in a primary care setting, studies on suspected acute bacterial conjunctivitis show that topical antibiotics improve the five day remission rate by only $31 \%$ compared with placebo. ${ }^{1}$ Therefore, in a primary care population, more than half of all patients with acute infectious conjunctivitis may receive unnecessary and not always effective antibiotic treatment. This prescription policy may increase the risk of antibiotic resistance, induce side effects, and lead to medicalisation and increases cost.

Can general practitioners differentiate between viral and bacterial conjunctivitis on the basis of signs and symptoms? Major ophthalmological textbooks list several signs and symptoms as being diagnostic for the cause of acute infectious conjunctivitis. The involvement of one eye, followed a few days later by the other eye, and the presence of an enlarged preauricular node are said to be signs indicating a viral cause. The involvement of the other eye within 24-48 hours is said to indicate a bacterial cause. A papillary or (pseudo)membranous conjunctivitis is suggestive of a bacterial origin, whereas a follicular conjunctivitis is said to suggest a viral origin. A mucopurulent or catarrhal discharge is said to be most commonly seen in bacterial or chlamydial conjunctivitis, whereas watery discharge is supposed to be more typical of a viral conjunctivitis. ${ }^{2-4}$ In most treatment trials on bacterial conjunctivitis the defined criteria for inclusion are purulent or mucopurulent discharge and conjunctival hyperaemia. How evidence based are these assertions? We planned a systematic review to assess the evidence on the diagnostic impact of these and other signs and symptoms.

\section{Participants, methods, and results}

We identified studies from PubMed, Embase, CINAHL, and the Cochrane Controlled Trials Register (issue 1,2002). We manually searched reference lists of relevant studies identified and of the guideline The Red Eye from the Dutch College of General Practitioners for additional studies. A consultant ophthalmologist supplied the names of commonly used textbooks, and we screened their bibliographies for additional studies. For PubMed and Embase we used search strategies devised for studies on diagnostic accuracy. We did the
Results of systematic search

\begin{tabular}{lccc} 
Stage & Action & Reviewer & No of citations selected \\
\hline 1 & Search $^{*}$ & RR & 6827 \\
\hline 2 & Duplicates and other topics removed & RR & 2903 \\
\hline 3 & Exclusion against criteria (see text) & RR and HW & 77 \\
\hline 4 & Exclusion on basis of full text & RR and HW & 1 \\
\hline
\end{tabular}

* Searches according to strategies devised for studies on diagnostic accuracy by Bachmann et al. J Am Med Inform Assoc 2002;9:653-4 and J Med Libr Assoc 2003;91:63-8.

searches in the first months of 2002 and limited them to studies in humans.

Studies were eligible for inclusion if they compared signs, symptoms, or both with the outcome of a bacterial culture. We excluded studies in neonates, postoperative (eye) patients, or trachoma and case studies, letters, and expert opinions.

After a thorough search and screening of 6872 references, we found one eligible study (table). ${ }^{5}$ However, on critical appraisal with the QUADAS instrument, this study seemed methodologically unsound.

\section{Comment}

We were unable to find evidence of the diagnostic usefulness of clinical signs, symptoms, or both in distinguishing bacterial conjunctivitis from viral conjunctivitis. Therefore, claims that certain signs and symptoms have diagnostic power, as cited in major textbooks and used in treatment trials to select patients, seem not to be based on evidence. Further research is needed to provide general practitioners with easy to use diagnostic tools to differentiate bacterial from viral conjunctivitis to tailor antibiotic prescriptions.

Contributors: HCPMvanW had the original idea. RPR did the search and collected the literature. HCPMvanW and RPR reviewed the collected literature. GterR designed the study. RPR wrote the first draft of the paper, which was edited by all other authors. PJEB is the guarantor for the study.

Funding: Dutch College of General Practitioners, Utrecht. Competing interests: None declared.

Ethical approval: Not needed.

1 Sheikh A, Hurwitz B. Topical antibiotics for acute bacterial conjunctivitis: a systematic review. BrJ Gen Pract 2001;51:473-7.

Krachmer JH. Cornea. St Louis: Mosby, 1997.

3 Tasman W,Jaeger EA. Duane's clinical ophthalmology on CD-ROM. Lippincot Williams \& Wilkins, 2001.

4 Kanski JJ. Clinical ophthalmology: a systematic approach. Oxford: Butterworth-Heinemann, 1999

5 Riva C, Perlino P, Valpreda A, Ricotti E, Castagneri G, Balbo L, et al. [Long lasting conjunctivitis: research of etiological factors]. Minerva Pediatr 1992;44:595-600. (In Italian.)

(Accepted 7 July 2003)

\section{Endpiece}

\section{Three sides}

There are three sides to every story-yours, mine, and the truth.

Robert Evans, actor and producer Health, Department of General Practice, Academic Medical Centre-University of Amsterdam, Meibergdreef 15, $1105 \mathrm{AZ}$,

Amsterdam,

Netherlands

Remco P Rietveld general practitioner Henk C P M van Weert

general practitioner Patrick J E Bindels professor in general practice

Horten-Zentrum, Universitätsspital Zürich, Postfach Nord, 8091 Zürich, Switzerland Gerben ter Riet epidemiologist

Correspondence to: R P Rietveld r.p.rietveld@ amc.uva.nl

BMJ 2003;327:789
Division of Clinical Methods and Public 